\section{SP0043 WORK RETENTION REHABILITATION IN PRACTICE}

Y. Prior ${ }^{1,2}$ on behalf of Rehabilitation Research Group, University of Salford. ${ }^{1}$ Health Sciences, University of Salford, Manchester; ${ }^{2}$ Rheumatology, Mid Cheshire NHS Trust Leighton Hospital, Crewe, United Kingdom

This session aims to meet health professionals' needs in recognising, examining, and identifying problems that patients with inflammatory arthritis (IA) face at work. We know that one in five people with inflammatory arthritis lose their job within five years of diagnosis and, once work disabled, they are unlikely to return to work. Therefore, preventive interventions targeted at work retention, which aim to help people to stay at work, are needed to avoid the transition from work instability to work disability. Rheumatology health professionals play an important role in identifying patients' work problems at an earlier stage and helping them to stay at work. Currently, there are no set pathways to outline health professionals' approach to identifying people with work problems and/or work rehabilitation interventions in the UK. Further, there is a need to train health professionals in the importance of the early recognition of these problems and evidence-based interventions to address work instability.

This session will focus specifically on the rheumatology occupational therapy-led work rehabilitation taken place in a NHS setting in the UK. Using real life case studies, Dr Prior will discuss the standardised assessments used in identifying work problems, collaborative goal setting and work interventions to provide a snapshot of work rehabilitation in the context of clinical practice.

Disclosure of Interest: None declared

DOI: 10.1136/annrheumdis-2017-eular.7240

\section{WEDNESDAY, 14 JUNE 2017 \\ Comorbidities: having one RMD is enough - we don't need anything else}

\section{SP0044 SCREENING FOR COMORBIDITIES IN DAILY PRACTICE: WHO AND HOW? \\ L. Gossec. Paris 06 University, Pitié-Salpétrière Hospital, Paris, France}

Comorbidities are frequent in RMDs, as in many other chronic diseases. In inflammatory rheumatic diseases, the most frequent comorbidities are cardiovascular diseases, depression, infections and cancers. Some of these diseases are more frequent than in persons without RMDs, some are not more frequent but are more often under-assessed and under-treated. These comorbidities and their risk factors need to be screened for. Screening in daily practice is not so simple since it necessitates time, physician expertise and patient cooperation. In this talk, we will address recent recommendations on how to screen for comorbidities, how often and in which setting this should be performed, and the respective roles in screening of rheumatologists, rheumatology nurses and persons with RMDs. Disclosure of Interest: None declared

DOI: 10.1136/annrheumdis-2017-eular.7135

\section{SP0045 DO PATIENT ORGANISATIONS HAVE A ROLE TO PLAY IN PREVENTING CO-MORBIDITIES?}

A.M. Bosworth. n/a, National Rheumatoid Arthritis Society, Maidenhead, United Kingdom

My talk will look in detail at how patient organisations can play a role in preventing co-morbidities. NRAS is currently engaged in two major projects which are both directly concerned with the prevention of co-morbidities. During RA Awareness Week in the UK, 19-26 June, NRAS will launch "Love your Heart" an on-line, interactive video programme to educate people with RA about their increased risk of heart disease and atherosclerosis. This programme explains in simple terms why people with RA are at increased risk of cardiovascular disease. The programme provides the opportunity to appraise individual risk factors, complete a QRISK2 assessment with their GP and provides a cognitive-behavioural framework to empower people to change their behaviours and achieve a healthier lifestyle, thereby reducing risk of heart disease. Baseline and 6 months evaluations are built into the programme. It has been developed with input from a consultant rheumatologist, GP, and other health experts as well as patients. If time permits I will also a describe a research study we are undertaking with 3 hospitals in the UK whereby we will randomly assign newly diagnosed patients to receive a targeted support fprogramme rom NRAS or be in a control group which does not receive this support for a period of 6 months. Our aim is to show that the group receiving the targetted support has less anxiety and depression, although other outcomes will also be measured.

Disclosure of Interest: None declared

DOI: 10.1136/annrheumdis-2017-eular.7230

\section{SP0046 PREVENTION AND THE PROTECTIVE ROLE OF EXERCISE AND LIFESTYLE INTERVENTIONS ON COMORBIDITIES IN RHEUMATIC DISEASES}

S. Garcia Diaz. Rheumatology, Consorci Sanitari Integral, Hospital Moises Broggi Sant Joan Despi, Sant Joan Despi, Spain

Background: It has been recognised that patients with rheumatic diseases are at increased risk of developing comorbid conditions such as cardiovascular disease (CVD), malignancies, infections and osteoporosis (among others). Lifestyle interventions (non smoking, non alcohol, healthy diet and exercise) may play an important role to reduce comorbidities in these patients.

Objective: To find if lifestyle interventions and especially exercise play a vital role in preventing comorbidities in rheumatic diseases through an extensive review of recent literature.

Disclosure of Interest: None declared

DOI: 10.1136/annrheumdis-2017-eular.7223

\section{THURSDAY, 15 JUNE 2017 Cytokines and chemokines}

\section{SP0047 TYPE I INTERFERON SYSTEM IN AUTOIMMUNITY}

L. Rönnblom. Department of Medical Sciences, Uppsala University, Uppsala, Sweden

The type I interferon (IFN) system is our main defense against viral infections and can be activated by a large number of sensors of nucleic acid, triggering the production of more than 15 different proteins with antiviral and immunostimulatory capacity. There are several observations suggesting an important role for this system in the etiopathogenesis of SLE and other autoimmune diseases. Among these are the reported development of autoimmune diseases during treatment with IFN-alpha, a prominent increase in the expression of type I IFN regulated genes (an IFN signature) in a number of rheumatic diseases, the existence of endogenous or self derived IFN inducers in SLE patients and a genetic association between autoimmune diseases and gene variants within the type I IFN signaling pathway.

The type I IFN system is closely connected to a number of cytokine and chemokine pathways, which all can contribute to both the IFN signature and the type I IFN effects. Important type I IFN effects are maturation and differentiation of dendritic cells, activation of $T$ and $B$ cells with enhanced antibody production and induction of increased expression of autoantigens. Consequently, type I IFNs can act as an immune adjuvant and promote an autoimmune process. Recent data have also shown that the regulation of the type I IFN system is abnormal in SLE, which all together suggests that inhibition of the type I IFN system could be beneficial in SLE and possible also other autoimmune diseases. Many different therapeutic targets exists and several studies are in progress aiming to block, or down-regulate, the activated type I IFN system in SLE. A number of studies with monoclonal anti-IFN-alpha antibodies have been reported, and a small study investigating vaccination with an interferon-alpha-kinoid against IFN-alpha has been published. Recently, a phase Ilb study targeting the type I IFN receptor in moderate to severe SLE was published, reporting substantially reduced disease activity. Other therapeutic possibilities include elimination of the endogenous IFN inducers and inhibition of key molecules in the type I IFN signaling pathway. The results so far shows that it's possible to suppress the IFN signature and improve several biomarkers in SLE patients without major safety problems. The challenge for the future is to modulate the interferon system in autoimmune diseases more precisely and realize that different treatments may be appropriate in various patients. Disclosure of Interest: L. Rönnblom Grant/research support from: AstraZeneca DOI: 10.1136/annrheumdis-2017-eular.7134

\section{SP0048 TARGETING OF ANGIOGENESIS}

S. Tas on behalf of European Synovitis Study Group. AMC Clinical Immunology \& Rheumatology, Amsterdam Rheumatology and immunology Center, Amsterdam, Netherlands

Angiogenesis is de novo capillary outgrowth from pre-existing blood vessels. This process not only is crucial for normal development, but also has an important role in supplying oxygen and nutrients to inflamed tissues, as well as in facilitating the migration of inflammatory cells to the synovium in rheumatoid arthritis, spondyloarthritis, and other tissues in systemic autoimmune diseases. Neovascularization is dependent on the balance of proangiogenic and antiangiogenic mediators, including growth factors, cytokines, chemokines, cell adhesion molecules and matrix metalloproteinases. In this lecture I will provide an overview of the various pathways that govern these angiogenic processes and discusses potential approaches to interfere with pathological angiogenesis, and thereby ameliorate inflammatory disease, by targeting these pathways specifically in endothelial cells.

Key Messages:

- In chronic inflammatory diseases, angiogenesis enables increased delivery of oxygen and nutrients to immune cell populations accumulating in inflamed tissues, and contributes to further immune cell infiltration 